\title{
Two-Photon Scanning Laser Ophthalmoscope
}

\author{
Tschackad Kamali, Spring RM. Farrell, \\ William H. Baldridge, Jörg Fischer, \\ and Balwantray C. Chauhan
}

\subsection{Introduction}

Two-photon excitation (TPE) fluorescence imaging is a powerful emerging tool in biomedical applications, providing high penetration depth and inherent three-dimensional (3-D) sectioning at the subcellular level [1-3]. The retina is the only tissue in which single neurons can be imaged optically and noninvasively due to the high transparency of the preretinal tissues $[4,5]$. TPE with infrared light (IR) is particularly well suited for

Portions of the text in this Chapter from 'Simultaneous in vivo confocal reflectance and two-photon retinal ganglion cell imaging based on a hollow core fiber platform. J Biomed Opt. 2018 Mar;23(9):1-4. doi: 10.1117/1. JBO.23.9.091405.' Reproduced under a Creative Commons Attribution 3.0 Attribution License (https://creativecommons.org/licenses/by/3.0/legalcode).

T. Kamali $(\bowtie) \cdot$ J. Fischer

Heidelberg Engineering GmbH, Heidelberg, Germany

\section{S. RM. Farrell}

Retina and Optic Nerve Research Laboratory,

Dalhousie University, Halifax, NS, Canada

Department of Physiology and Biophysics, Dalhousie University, Halifax, NS, Canada

Department of Medical Neurosciences, Dalhousie University, Halifax, NS, Canada

W. H. Baldridge

Retina and Optic Nerve Research Laboratory,

Dalhousie University, Halifax, NS, Canada in vivo retinal imaging. Reporter molecules in cell bodies can be excited with IR light to allow differential activation of rod and cone photoreceptors by wavelengths in the visually sensitive range to evoke responses in the retina [6-9]. An additional advantage of TPE is reduced phototoxicity [8]. TPE fluorescence imaging enables the study of functional physiological processes, which, in combination with in vivo ophthalmoscopy, represent powerful imaging techniques that are well suited for noninvasive in vivo retinal imaging. Applications include longitudinal tracking of disease progression, for example, in optic neuropathies in which retinal ganglion cells (RGCs), the output neurons from the eye to the brain, are lost.

Department of Medical Neurosciences, Dalhousie University, Halifax, NS, Canada

Department of Ophthalmology and Visual Sciences, Dalhousie University, Halifax, NS, Canada

B. C. Chauhan

Retina and Optic Nerve Research Laboratory,

Dalhousie University, Halifax, NS, Canada

Department of Physiology and Biophysics, Dalhousie University, Halifax, NS, Canada

Department of Ophthalmology and Visual Sciences, Dalhousie University, Halifax, NS, Canada 


\subsubsection{Retinal Signaling}

The retina is a photosensitive neuronal tissue that transmits visual information to the brain. Light, captured and focused by the eye, is converted from individual photons to an electrical signal that is propagated to the brain by action potentials. In the photoreceptors, isomerization of retinal is catalyzed by photons of incident light, which initiates a series of intracellular signaling events that converts the light stimulus into a chemical signal that is propagated through interneurons (horizontal cells, bipolar cells and amacrine cells) to the retinal ganglion cells (RGCs, Fig. 9.1) [10]. RGCs receive input from multiple bipolar and amacrine cells and encode these signals prior to transmitting this information to the brain via the optic nerve. Competing excitatory and inhibitory stimuli detected within an RGC's dendritic field are integrated to determine a net increase, decrease or null change in action potential firing. Much of this RGC processing generates visual acuity and contrast sensitivity [11]. There are many subtypes of RGCs with varying functional properties in the retina, which have been characterized at the single cell level ex vivo [12]; however, in vivo functional analysis of individual RGCs is lacking.

\subsubsection{Imaging Retinal Neurons}

Numerous studies have introduced fluorescent tracer molecules into RGCs to enable the visualization of retinal cells in experimental models, ex vivo [13]. These studies have provided an immense body of knowledge about the mammalian retina, including RGC densities, quantification of axonal transport and cell loss after axonal injury. However, these studies are cross-sectional in nature; requiring removal of the retina, histologic preparation and microscopic examination. In vivo confocal scanning laser ophthalmoscopy (CSLO) established a new longitudinal imaging paradigm that allows individual RGCs labelled with fluorescent reporter molecules (Table 9.1) in the rodent retina to be followed over time [13-
17] (Fig. 9.2). These studies (among countless others) have provided invaluable insights into the progression of dendritic retraction and RGC death after injury; however, inferring information about retinal function (or dysfunction) from structural imaging remains a challenge. Expression of genetically encoded calcium indicators (GECIs), such as GCaMP, in RGCs allows the visualization of neuronal activity [18]. The Thy1-GCaMP3 transgenic mouse line selectively expresses GCaMP in the majority of RGCs [19], which allows a subset of the RGC population to be studied over time. Changes in intracellular calcium levels during action potentials are reported by changes in GCaMP fluorescence, which can be imaged in vivo using modified CSLO systems [20]. One challenge that presents itself with in vivo functional imaging with CSLO is the overlap in spectral sensitivities of mammalian photoreceptors and the most commonly used fluorescent reporter molecules (Fig. 9.3 and Table 9.1). Murine experimental models can be used, where excitation of UV sensitive cones stimulates the visual pathway and differential excitation of the reporter molecule is possible, allowing for the visualization of functional responses in RGCs (Fig. 9.4). However, this method examines only a portion of the visual system and further, humans do not have UV wavelength sensitive cones, making this approach unlikely for clinical translation. TPE addresses both of these limitations by using brief pulses of IR wavelength light, which is able to penetrate the preretinal tissues and excite fluorophores without stimulating the photoreceptors (Fig. 9.5 and Table 9.1).

Studies have developed TPE imaging systems to examine functional responses of RGCs in vivo $[9,21]$. In TPE imaging, pulses of long wavelength light (typically in the IR range), approximately double the single photon excitation wavelength (Table 9.1) [22], are used to create conditions where two photons simultaneously stimulate the fluorescent reporter molecule to create energy similar to that of a single short wavelength photon (detailed theory in Sect. 9.2). Yin et al. [21] used adaptive optics 


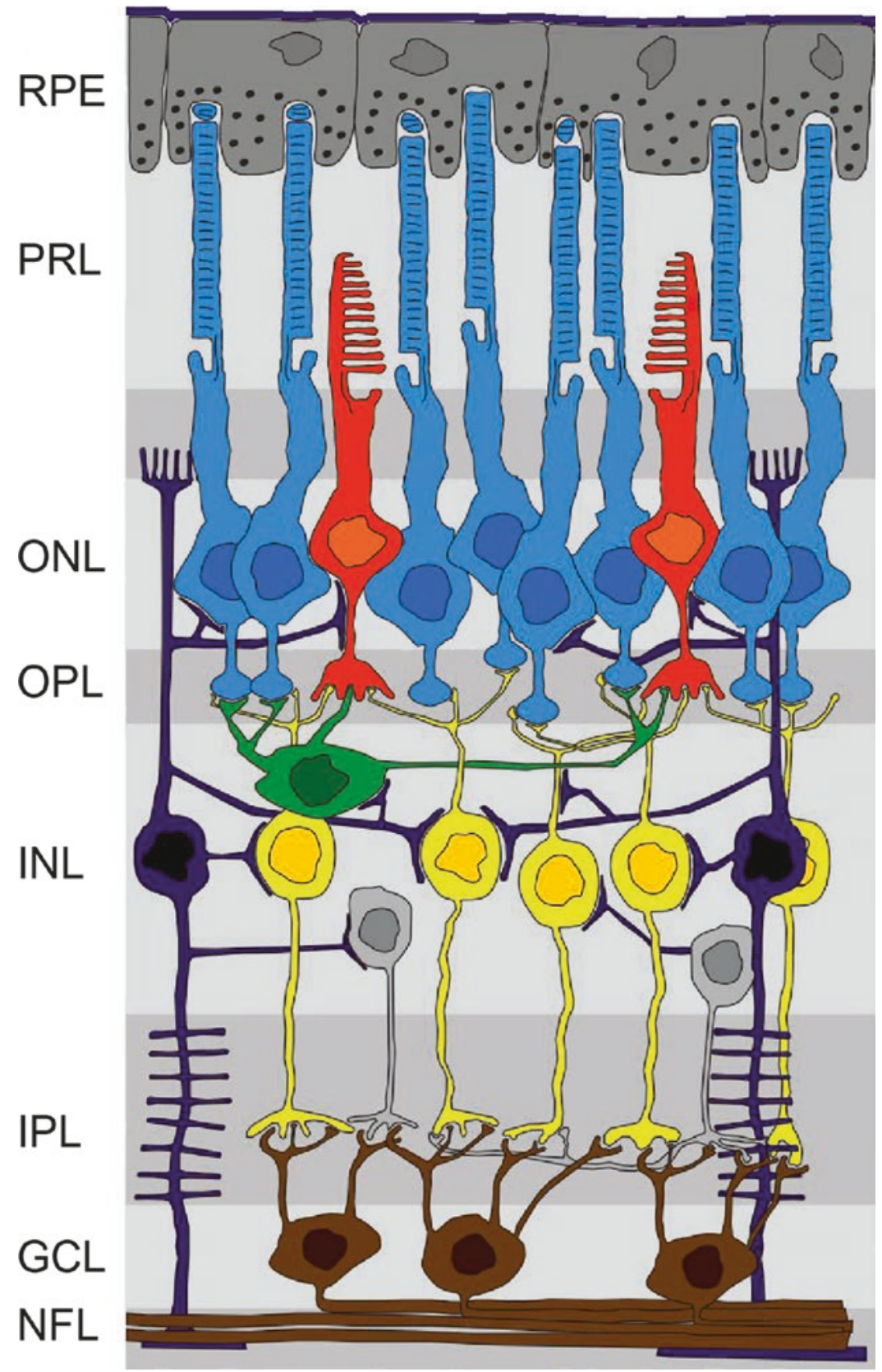

Fig. 9.1 Schematic representation of cell types shown in a retinal cross section. Incident light, focused on the retina through the lens, travels through the inner layers of the retina to reach the photoreceptors (PRs, rods: blue; cones: red), where visual pathway signaling begins. The signal is propagated from photoreceptors to bipolar cells (BPCs, yellow) to retinal ganglion cells (RGCs, brown), which transmit visual information to the brain via axons that make up the nerve fiber layer (NFL). Horizontal cells (HCs, green) and amacrine cells (ACs, grey) make lateral connections, which modulate the signal and Müller glia cells (dark purple) act as metabolic support cells in the retina. $R P E$ retinal pigment epithelium, $P R L$ photoreceptor layer, $O N L$ outer nuclear layer, $O P L$ outer plexiform layer, $I N L$ inner nuclear layer, $I P L$ inner plexiform layer, $G C L$ ganglion cell layer, $N F L$ nerve fiber layer. Modified from: Hartpete (https://commons.wikimedia.org/wiki/ File:Retina.jpg) [35], "Retina", labels modified by $S$ Farrell 2018, https://creativecommons.org/licenses/ by-sa/3.0/legalcode 
Table 9.1 Exogenous fluorophores commonly used as reporters to image retinal neurons

\begin{tabular}{l|l|l}
\hline & $\begin{array}{l}\text { Single photon } \\
\text { excitation }(\mathrm{nm})\end{array}$ & $\begin{array}{l}\text { Two photon } \\
\text { excitation }(\mathrm{nm})\end{array}$ \\
\hline $\begin{array}{l}\text { Small molecule dyes } \\
\begin{array}{l}\text { Fura-2 }\left(\mathrm{Ca}^{2+}\right. \\
\text { bound/Ca } \\
\text { unbound }\end{array}\end{array}$ & $335 / 363$ & 780 \\
\hline Fluo-3 & \multicolumn{2}{l}{} \\
\hline Fluorescein (FITC) & 494 & 810 \\
\hline Fluorescent proteins & 436 & 780 \\
\hline CFP & 436 & 850 \\
\hline GFP & 491 & 970 \\
\hline YFP & 490 & 970 \\
\hline GCaMP & \multicolumn{2}{|l}{} \\
\hline
\end{tabular}

Data from: [28-30]
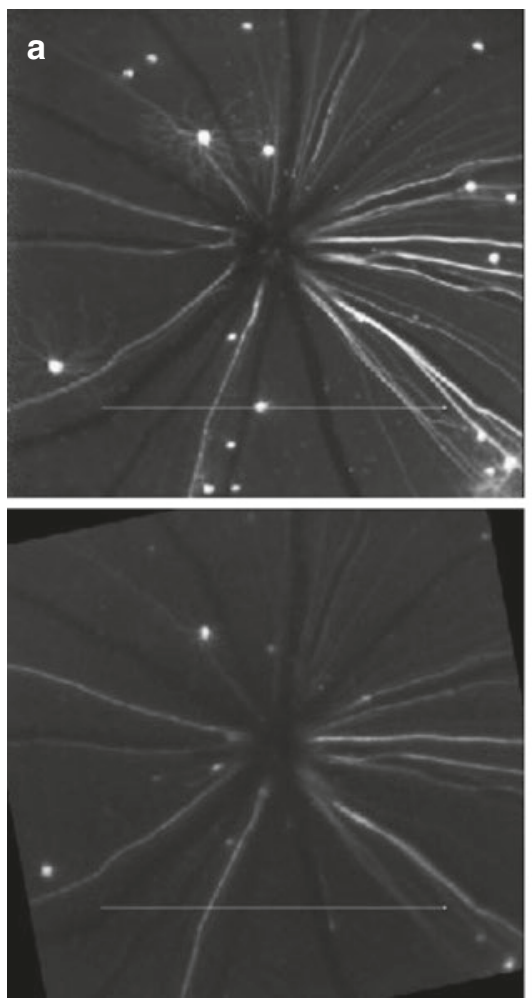

Fig. 9.2 Longitudinal in vivo CSLO images of fluorescent reporter molecules expressed in RGCs of the murine retina before and after optic nerve transection (ONT). (a) Transgenic Thy 1-YFP line $\mathrm{H}$ mice express YFP in $<0.5 \%$ of RGCs. Top: Baseline in vivo imaging shows sparse labeling of a few RGCs, including their dendrites and axons extending to the optic nerve head. Bottom: Follow-up imaging of the same subject, in the same retinal
(AO, described in Chap. 16) in conjunction with TPE to image RGC activity, reported by GCaMP5, in non-human primate retina. Similarly, but without the use of AO, Bar-Noam et al. [9] measured in vivo changes in GCaMP6 fluorescence in RGCs, in response to stimulation of the visual pathway. This study demonstrated in vivo calcium transients in RGCs similar to those reported previously in ex vivo retinal preparations [12]. Together, these studies provide a foundation to further develop TPE as an imaging platform to study RGC function in vivo; however, clinical translation requires further refinement.
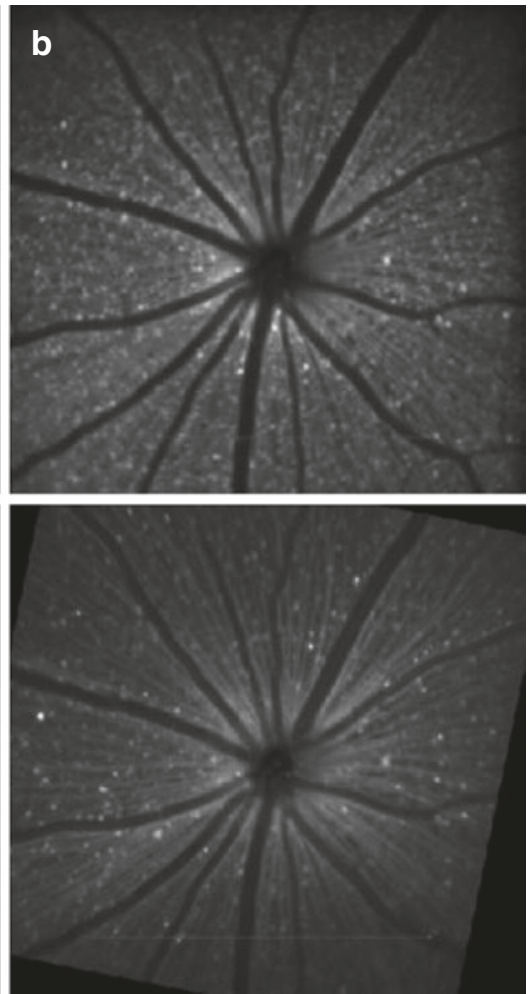

location, 7 days post-ONT shows fewer YFP labelled RGCs and retraction of remaining RGC dendrites. (b) Transgenic Thy1-GCaMP3 mice express GCaMP3 in approximately $65 \%$ of RGCs. Top: Baseline imaging shows many labelled RGCs, including axons. Bottom: 7 days post-ONT, dramatic RGC loss can be observed. All images show a $30^{\circ}$ field of view 
Fig. 9.3 Photoreceptor spectral sensitivities in mouse and human retina. Cone photoreceptor wavelength sensitivities in Human (a) and Mouse (b) retinas. Note that single photon excitation wavelengths of common reporter molecules (Tables 9.1 and 9.2) are within these ranges of photoreceptor activation, while the TPE excitation wavelengths used to stimulate these reporters (Tables 9.1 and 9.2) are outside the spectral sensitivities of all photoreceptor types. Modified from: [36]. Reproduced under Creative Commons Attribution License (CC BY; https:// creativecommons.org/ licenses/)
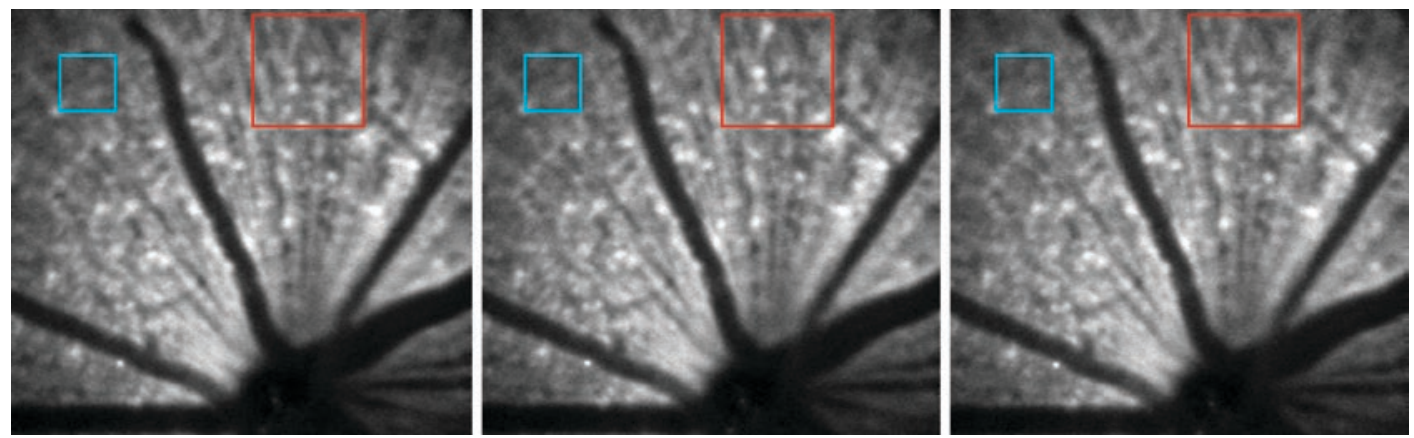

Fig. 9.4 In vivo CSLO images of calcium responses in RGCs, reported by GCaMP3 in the Thy1-GCaMP3 mouse retina. In vivo single photon CSLO images taken with the Spectralis Multiline. Left: baseline GCaMP3 fluorescence (488 nm excitation). Middle: UV (365 nm) light-evoked

\subsubsection{Imaging Other Retinal Cell Types In Vivo}

Several endogenously expressed molecules have been found to exhibit fluorescence and lend themselves to act as reporter molecules response of the visual pathway increased GCaMP3 fluorescence in some cells (red box), while other cells decreased fluorescence during UV stimulation (blue box). Right: post-light stimulus GCaMP3 fluorescence. Each image reflects the integration of approximately $25 \mathrm{~s}$ of recording

(Table 9.2), but require single photon excitation by UV wavelengths, which are absorbed by the cornea and lens and also have phototoxic effects in the human retina. Using TPE, these endogenous fluorophores can be excited in vivo and act as endogenous reporter molecules. Recent 


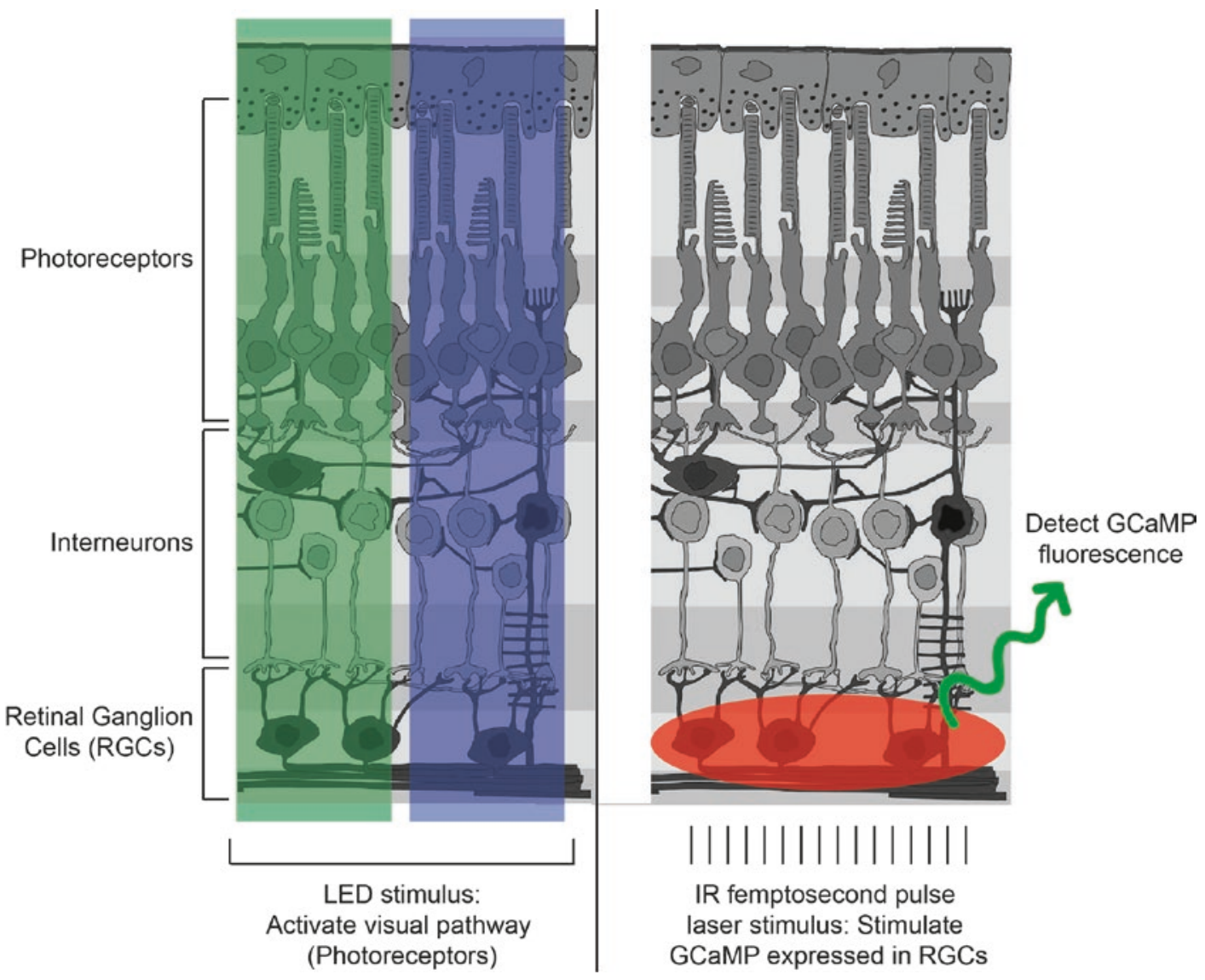

Fig. 9.5 Schematic representation of differential activation of the visual pathway and excitation of GCaMP. Left: Flashes of LED light stimulate photoreceptors (S- and M-cones, blue and green light, respectively) and activate the visual pathway. Right: In between LED flashes, the IR femtosecond pulse laser $(930 \mathrm{~nm})$ stimulates GCaMP3 in

Table 9.2 Endogenous fluorophores expressed in retinal cells

\begin{tabular}{l|l|l|l}
\hline & $\begin{array}{l}\text { Single } \\
\text { photon } \\
\text { excitation } \\
(\mathrm{nm})\end{array}$ & $\begin{array}{l}\text { Two photon } \\
\text { excitation } \\
(\mathrm{nm})\end{array}$ & $\begin{array}{l}\text { Fluorescence } \\
\text { lifetime }(\mathrm{ns})\end{array}$ \\
\hline $\begin{array}{l}\text { NAD(P)H } \\
\text { (free/protein } \\
\text { bound) }\end{array}$ & $300-380$ & 750 & $0.3 / 2.0-2.3$ \\
\hline $\begin{array}{l}\text { FAD (free/ } \\
\text { protein } \\
\text { bound) }\end{array}$ & $420-500$ & 900 & $2.91 /<0.01$ \\
\hline Collagen & $280-350$ & 880 & $<5.3$ \\
\hline Lipofuscin & $340-500$ & 800 & $<2.2$ \\
\hline
\end{tabular}

Data from: [31-34]

${ }^{a}$ Examples for demonstration purposes, other excitation wavelengths are possible, due to the presence of multiple peaks and broad TPE spectra of endogenous molecules the RGC layer. Due to the $2 \mathrm{P}$ effect, localizing laser energy in the RGC layer, photoreceptors are not be activated by the IR laser, thus effectively separating visual pathway and reporter excitation. Modified from: [35], "Retina", labels and colour modified by S Farrell 2018, https://creativecommons.org/licenses/by-sa/3.0/legalcode

studies have imaged retinal structures of the inner and outer retina, with single cell resolution, using AO enhanced TPE [23-27]. In addition to gaining structural insights, fluorescence lifetime information, can be extracted from these data. Changes in fluorescence lifetime (described in Chap. 10) of endogenous molecules, such as NAD(P)H and FAD, has been suggested as a functional measure of cellular activity and health in the retina [26]. Thus, together, TPE and fluorescence lifetime measurements may provide functional data from the retina without the use of exogenous reporter molecules, which may allow tracking of disease progression in patients. 


\subsection{Theoretical Background}

\subsubsection{Luminescence, SPA and TPA}

Electronic excitation of molecules can be performed either by a physical (absorption of light), chemical or mechanical process. Luminescence describes the effect of a molecule emitting light after de-excitation into the ground state. If this excitation was created by the absorption of photons, then it is called photoluminescence. Photoluminescence of molecules can be divided into two groups, fluorescence and phosphorescence, differing in electronic configuration in the excited state and emission pathways [37].

Fluorescence is the ability of some atoms and molecules to absorb photons with a particular energy and to re-emit photons with reduced energy (red shifted) after a short time interval in the nanosecond time scale, referred to as fluorescence life time. Phosphorescence differs from fluorescence by the electronic transition pathway (intersystem crossing) into the excited triplet state resulting in a much longer excited state lifetime in the range of milliseconds to hundreds of seconds [38].

Molecules can transit from the ground state (lower energy) to the excited state (higher energy) by absorbing photons with an energy being at least equal to the energy difference between the excited and ground state. This electronic excitation can be achieved either by linear or nonlinear photon absorption. Linear excitation of the molecule is achieved by single photon absorption (SPA) whereas nonlinear absorption describes the case when two or more photons with less energy (as compared to the single photon excita- tion) combine to bridge the energy gap needed to excite the atom or molecule. Most widely used nonlinear excitation in biomedical research is two-photon absorption (TPA), where two photons with half the energy of SPA combine for electronic excitation of the molecule [39].

From the electronic excited state the molecule can return to the electronic ground state either by non-radiative relaxation, by emitting a photon with longer wavelength (fluorescence) or by phosphorescence after intersystem crossing. All three phenomena are depicted in Fig. 9.6.

Combination of confocal scanning laser microscopy (CSLM) with SPA or TPA allows one to generate two-dimensional fluorescence images of the specimen under investigation. Within the next sections, CSLM with SPA will be termed linear fluorescence imaging (LFI) and CSLM with TPA will be termed two-photon excitation fluorescence imaging (TPEFI).

\subsubsection{TPA Probability and Dependencies}

The probability for a two-photon absorption (TPA) process to occur, is dependent on the physical properties of the molecule (termed as TPA cross-section $\left.\left(\sigma_{2}\right)\right)$ and the spatiotemporal properties of the excitation light. TPA requires the "simultaneous" arrival of two photons (time interval within $10^{-18} \mathrm{~s}$ ) wherefore it has a quadratic dependency on the average incident light power $\left(P_{\text {avg }}\right)$, making it a nonlinear process. Since the TPA cross sections are usually very low as compared to SPA cross sections [40, 41], tempo-
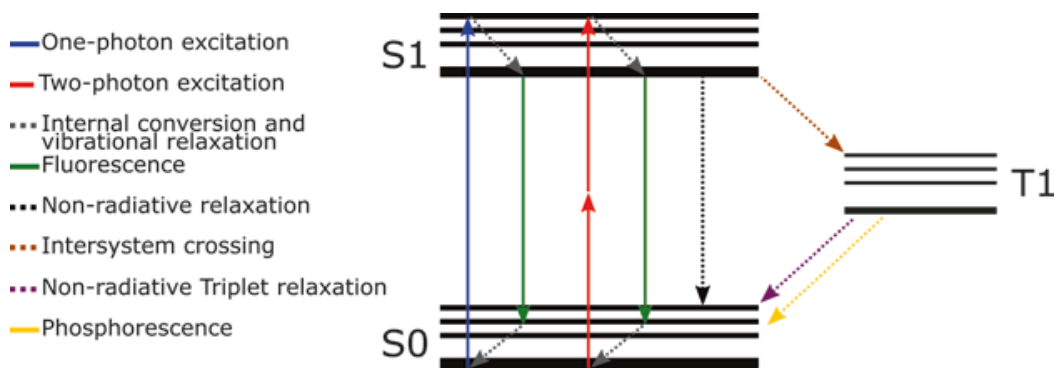

Fig. 9.6 Jablonski Diagram depicting one-photon excitation fluorescence, two-photon excitation fluorescence, internal conversion and vibrational relaxation, non-radiative relaxation, intersystem crossing and phosphorescence 
ral and spatial confinement is crucial for increasing TPA signal generation.

Temporal confinement is achieved by the use of pulsed laser sources $\left(f_{p}\right)$ with pulse durations $\left(\tau_{p}\right)$ below a few pico-seconds, resulting in high peak powers. The use of pulsed laser sources as compared to continous wave lasers enhances the signal by a factor of $\sim 1 / \tau_{p} f_{p}$, which would lead to a signal enhancement of $10^{5}$ by using commonly available femtosecond laser sources with pulse durations of $100 \mathrm{fs}$ and pulse repetition rates of $100 \mathrm{MHz}$.

Spatial concentration is dependent on the excitation wavelength $\left(\lambda_{\text {exc }}\right)$ and high numerical aperture (NA) objectives, leading to a small focal volume resulting in high peak intensities.

The probability, $n_{a}$, for two-photons to be absorbed simultaneously (while neglecting saturation effects and considering paraxial optics) at the focal plane per laser pulse and per fluorophore can be written as [42]:

$$
n_{a}=\sigma_{2} \frac{P_{a v g}^{2}}{\tau_{p} f_{p}^{2}}\left(\pi \frac{N A^{2}}{h c \lambda_{\text {exc }}}\right)^{2}
$$

where

$h$ is Planck's constant

$c$ is the speed of light

As can be seen from the formula above the laser pulse duration is inversely proportional to the twophoton signal generation, resulting in increased two-photon signal generation when reducing the laser pulse duration. In practice however, short pulses lead to higher dispersion which have to be compensated. Dispersion is a phenomenon in which the phase velocity of a wave is coupled to its frequency resulting in wavelength-dependent refractive indices in optical media. The temporal profile of the laser pulse is directly related to its spectral bandwidth and therefore shorter pulses are more vulnerable to dispersion effects leading to broadened pulses and less efficient two-photon excitation if not compensated $[4,43]$.

\subsubsection{Optical Resolution}

In linear fluorescence imaging, fluorescence photons are also generated above and below the focal plane, wherefore axial sectioning can be enhanced by spatially filtering the emitted fluorescence signal at the detection plane. Even though axial resolution is improved by reducing the pinhole diameter, fluorescence yield is also reduced, since fluorescence photons suffering from chromatic aberrations and strong scattering are blocked and cannot reach the detector [22]. Axial and lateral confinement in TPEFI on the other hand is an intrinsic property of the nonlinear excitation process neglecting the need for spatially filtering the signal by a pinhole.

In TPEFI the effective point spread function $\left(P S F_{T P}\right)$ can be described by the square of the illumination $P S F_{\text {ill }}$ [42]:

$$
P S F_{T P}=\left(P S F_{i l l}\right)^{2} \approx P S F^{2}\left(\frac{v}{2}, \frac{u}{2}\right)
$$

with $v=k(N A) r, u=k(N A)^{2} z$ and assuming $\lambda_{\text {ill }} / 2 \approx \lambda_{f}$, v/2 and $\mathrm{u} / 2$ denoting an about doubled illumination wavelength in contrast to singlephoton excitation.

Considering full illumination of the back aperture of the microscope objective (beam diameter $>$ back aperture diameter) the diffraction limited resolution for TPEFI can be approximated by the Full Width at Half Maximum (FWHM) of a Gaussian fit to the squared illumination PSF. Lateral $(\Delta r)$ and axial fitted $(\Delta z)$ squared intensity PSF profiles are described as follows [37, 38]:

$$
\begin{gathered}
\Delta r=\left\{\begin{array}{l}
\frac{0.320 \sqrt{2 \ln 2} \lambda}{N A}, N A \leq 0.7 \\
\frac{0.325 \sqrt{2 \ln 2} \lambda}{N A^{0.91}}, N A \geq 0.7
\end{array}\right. \\
\Delta z=0.532 \sqrt{2 \ln 2 \lambda}\left(\frac{1}{n-\sqrt{n^{2}-N A^{2}}}\right),
\end{gathered}
$$

where NA is the numerical aperture of the objective lens, $\lambda$ the excitation wavelength and $n$ denoting the refractive index of the immersion medium.

At first sight the theoretical lateral and axial resolution of TPEFI seems worse than the case of LFI due to the longer wavelength being used for excitation. In practice however, the spatial resolu- 
tion of LFI and TPEFI that can be achieved are similar due to the use of finite-sized pinholes which broaden the theoretical PSF in LFI [22, 42].

\subsubsection{Linear SPA vs. Nonlinear TPA Imaging}

Linear fluorescence imaging (LFI) by SPA confocal scanning laser microscopy (CSLM) generates fluorescence over the entire excitation light cone (Fig. 9.7a), wherefore nonlinear imaging generates photons only in the vicinity of the focal spot (Fig. 9.7b).

In nonlinear imaging, in particular TPEFI two excitation, photons combine their quantum energies and generate a photon with higher quantum energy which leads to a "bluer" emission as compared to the excitation. This is different from the red shifted emission occurring in LFI, and allows the use of excitation light in the near-infrared (NIR) wavelength range (700-1000 nm) for commonly used fluorescent markers emitting in the visible spectral range [32, 39].

The use of longer wavelengths in TPEFI allows the excitation light to penetrate deeper into scattering tissue, since longer wavelengths exhibit less scattering, and also phototoxic effects are reduced, since less one-photon endogenous

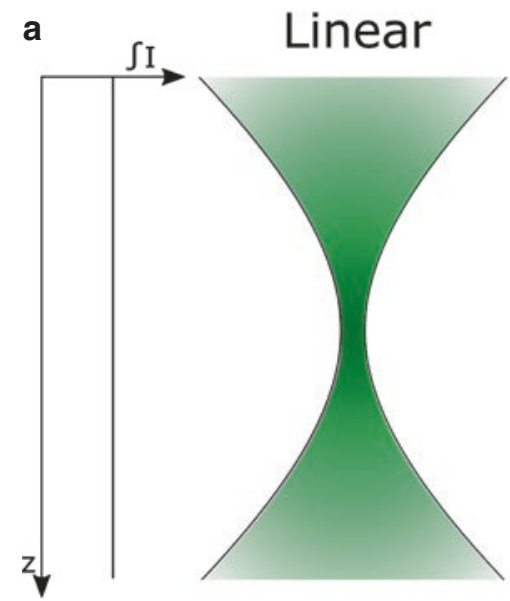

Fig. 9.7 In single photon fluorescence imaging, the linear relation between the incident and emitted photon leads to fluorescence generation over the entire light illumination cone (a), in two-photon excitation fluorescence imag- absorbers are available at this wavelength [22, 44]. Another major advantage of nonlinear imaging is the nonlinear dependence of the signal intensity (S) to the excitation light intensity (I) which is quadratic for TPEFI $\left(S \propto I^{n}\right)$. This quadratic dependency allows the TPA to occur only at the focal volume and its close vicinity (spatially confined excitation) when focusing the laser beam through a microscope. As can be seen in Fig. 9.7b, no TPA fluorescence is created in planes above or below the focal volume which differs from SPA fluorescence, where fluorescence is created over the entire depth of the excitation light cone. The lack of out of focus TPA fluorescence provides advantages for long term in vivo imaging of biological tissue, since tissue viability is enhanced by reduced photo damage $[22,44,45]$. TPEFI further provides inherent three-dimensional sectioning capabilities without the need of spatially filtering the emitted light by the use of a confocal pinhole as it is the case for LFI.

Even in strongly scattering media this inherent sectioning capability is maintained, because the density of scattered excitation photons are usually too low for nonlinear signal generation which is important for deep imaging, since all the signal photons reaching the detector originated from the focal volume and its vicinity carrying useful information [2, 22, 42]. However, special care

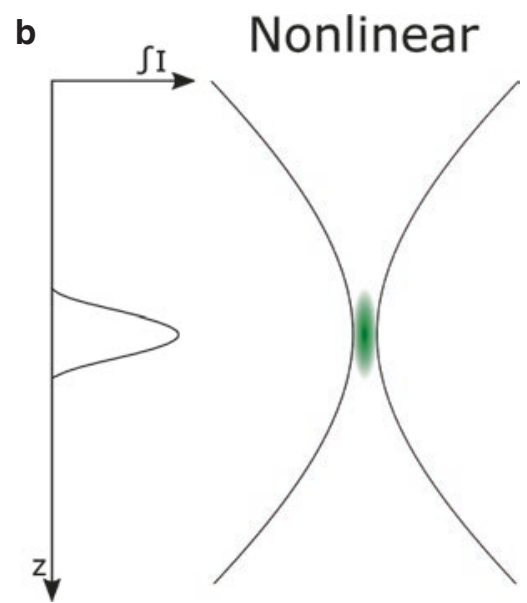

ing, fluorescence is only generated near the focal plane due to the nonlinear relationship between the signal- and illumination-intensity (b) 
has to be taken by choosing the collection optics for optimizing the fluorescence for deep imaging, because of the increased spatio-angular space due to scattering.

\subsection{Experimental Setup and Results}

From [46] Reproduced under Creative Commons Attribution License (CC BY; https://creativecommons.org/licenses/by/3.0/legalcode).

In this work, we demonstrate, for the first time to our knowledge, a system based on a hollow core fiber (HCF) that is capable of simultaneous in vivo confocal reflectance and two-photon imaging of RGCs through the mouse pupil without the use of $\mathrm{AO}$. One strategy to reduce the need for $\mathrm{AO}$ in the device presented here is the use of eye tracking software (Heidelberg Engineering, Heidelberg, Germany) that is currently implemented in clinical confocal scanning laser devices for ophthalmoscopy and tomography [47]. The real-time eye tracking software enables prolonged signal collection from the same spot, which is critical at low signal levels and in suboptimal light focusing conditions. The simultaneous acquisition of confocal reflectance and two-photon images colocalizes precisely the retinal location, where the two-photon recordings originate. Together, these features provide images with single cell resolution along with wider field fundus images. The use of an HCF for laser delivery allows our system to be split into a compact application unit (dashed blue area, Fig. 9.8) and a separate laser unit that can be placed on a nearby optical bench (gray shaded area, Fig. 9.8) without introducing noticeable pulse broadening, which is limited to approximately $-200 \mathrm{fs}^{2} / \mathrm{m}$ in our system. The application unit itself consists of a modified commercial scanning laser ophthalmoscope and an optical coherence tomography unit (Spectralis, Heidelberg Engineering) used routinely in clinical practice.

The Ti:Sapphire light source (Chameleon Ultra II, Coherent, Santa Clara, California) was tuned to a center wavelength of $930 \mathrm{~nm}$ for confocal reflectance imaging as well as two-photon signal generation. The laser had a repetition rate of $80 \mathrm{MHz}$ and produced pulses of 140 fs duration with an output power of $1.6 \mathrm{~W}$ at $930 \mathrm{~nm}$. A half-wave plate (HWP) and a polarization beam splitter were used for power adjustment. The group delay dispersion of the complete optical setup amounted to $\sim 7000 \mathrm{fs}^{2}$, which was compensated for by a femtosecond pulse compressor based on prism pairs (FSPC, Thorlabs, Newton, New Jersey). The pulse duration at the sample position was measured with an autocorrelator (Mini USB PMT NIR, APE, Berlin, Germany), which confirmed approximately transform-limited pulses of $154 \mathrm{fs}$. A CCD camera (FireflyMV, FLIR, Wilsonville, Oregon) was used to measure the beam profile at the sample position. Both second-order autocorrelation measurement and the Gaussian beam profile measurements are shown in Fig. 9.9.

After dispersion compensation, the laser beam was coupled to a 2-m HCF (GLOphotonics, Limoges, France) with a 45-mm focal length achromatic lens (AC254-045-B, Thorlabs) with which a coupling efficiency of $\sim 89 \%$ was achieved. The coupling lens was mounted on a 25-mm XYZ translation stage (PT3, Thorlabs) and the fiber on a three-axis microblock stage (MBT616D, Thorlabs).

The fiber output was coupled via FC connector to the fiber adapter plate that was mounted to the camera head of the modified Spectralis unit. The divergence of the output beam from the fiber was increased with a $-6.0-\mathrm{mm}$ focal length, biconcave, negative lens (LD2746-B, Thorlabs) to avoid the use of a longer focal length collimator before it was collimated with an achromatic doublet lens with a focal length of $25 \mathrm{~mm}$ (AC 127-025-B, Thorlabs). Two customized lens pairs of equal focal length, $\mathrm{f}=20 \mathrm{~mm}$ (L4, L5 in Fig. 9.8), were integrated to enable finer focus adjustment in the axial plane. Horizontal and vertical beam scanning was performed with the standard Spectralis scan unit. In combination with a customized 50-mm focal length scan lens, an intermediate image field of $5 \times 5 \mathrm{~mm}^{2}$ was produced. A customized 16-mm focal length tube lens translated the intermediate image field to a field of view of $\sim 17.5^{\circ}$ while achieving a beam size of $\sim 2.2 \mathrm{~mm}$ (overfilling the dilated mouse pupil). Both reflectance and two-photon fluores- 


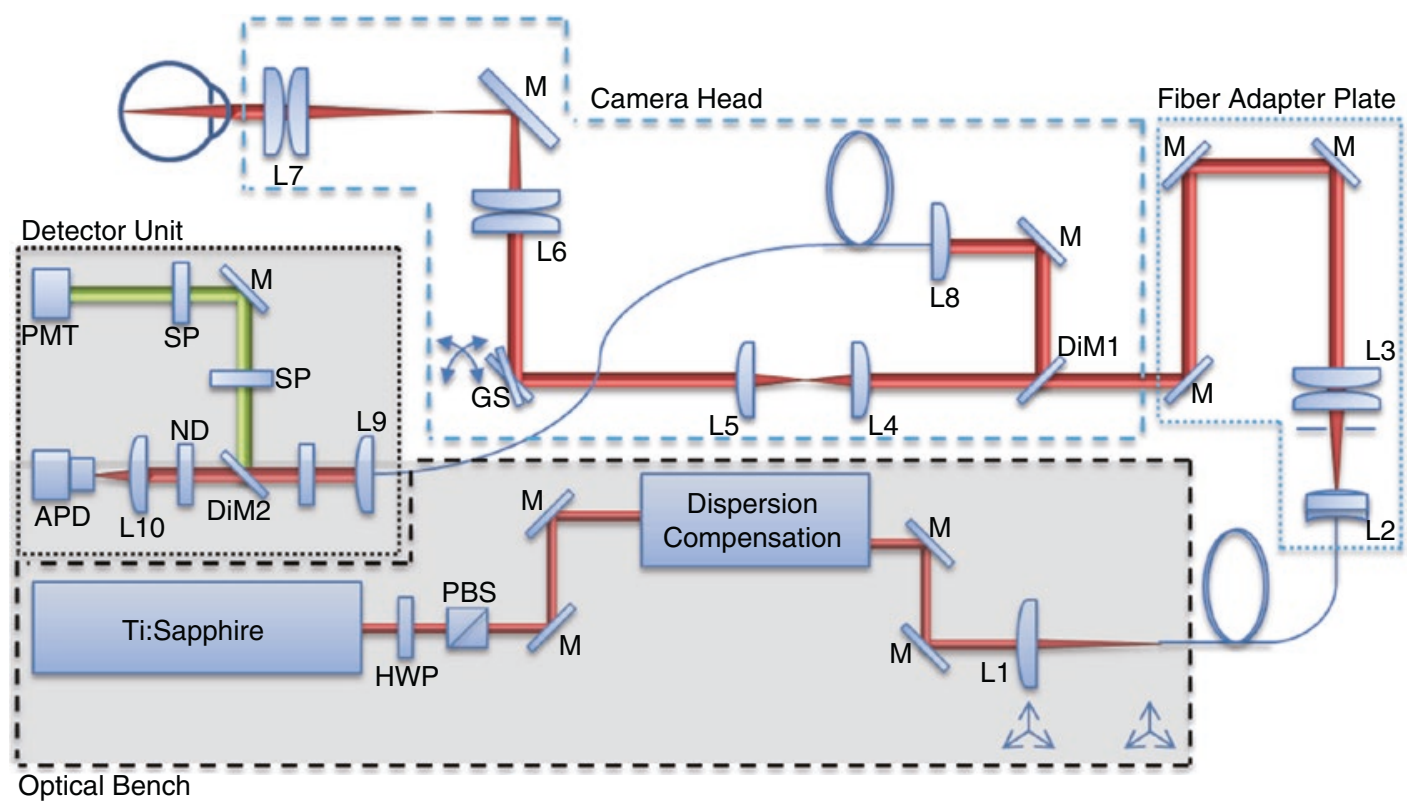

Fig. 9.8 Simultaneous confocal reflectance and twophoton imaging setup: the output of the Ti:Sapphire laser is adjusted with an HWP and a polarizing beam splitter. After dispersion compensation with prism pairs, the light is coupled into an HCF with a lens (L1), where both the $\mathrm{HCF}$ and L1 are mounted on separate three-dimensional translation stages. The output side of the fiber is connected via an FC connector to the fiber adapter plate (blue dotted line) that is attached to the modified Heidelberg Engineering Spectralis camera head (blue dashed line). The divergence of the fiber output is increased with a negative lens (L2) before coupling it to a double achromatic lens (L3). Two lenses of equal focal length (L4 and L5) were used for fine focal readjustments. Lateral scanning was performed with galvanometric scanners (GS), where the pivot point was imaged on the mouse pupil with a scan and tube lens (L6 and L7, respectively). A dichroic

cence signal were repassed through the scanning unit, resulting in a stationary, descanned light beam. A dichroic mirror (FF735-Di02, Semrock, Rochester, New York) was used to couple the fluorescence and reflectance signal into the detection branch, consisting of an 40-mm focal length achromatic lens and a $100-\mu \mathrm{m}$ multimode fiber, which guides the signal light to the external detection unit. The fiber output was collimated with a 12-mm focal length lens and a second dichroic mirror (FF735-Di02, Semrock) separated the visible fluorescence light from the near infrared (NIR) reflectance light. The reflectance light was further attenuated with a neutral density beam splitter (DIM1) in the camera head reflected the signal that was coupled to a multimode fiber with a lens (L8). In the detection unit (black dotted line), the output of the fiber was collimated with a lens (L9), and a dichroic mirror (DIM2) separated the fluorescence signal from the reflectance signal. In the reflectance path, an ND filter is used for attenuation. A lens (L10) focuses the light on an avalanche photodetector. The fluorescence signal path contains another short pass blocking filter (SP) before detection with a photomultiplier tube. Steering mirrors (M); shaded box indicates optical components placed on an optical bench; black dashed line represents the excitation path while black dotted line represents the detection path. From [46] Reproduced under Creative Commons Attribution License (CC BY; https://creativecommons.org/ licenses/ by/3.0/legalcode)

(ND) filter and focused on an avalanche photodiode (RCA, New York City) with a 20-mm focal length customized achromatic lens. In the fluorescence signal path, a short-pass filter (FF01$720 /$ SP-25, Semrock) was used to remove any leakage from the excitation light before being focused by a $100-\mathrm{mm}$ focal length achromatic lens (47-972, Edmund Optics, Barrington, New Jersey) onto the photon counting detector (HPM100-50, Becker-Hickl, Berlin, Germany) connected to a time-correlated single photon counting (TCSPC) module (SPC-150, BeckerHickl). All imaging was performed with a horizontal line scan rate of $8 \mathrm{kHz}$ and a pixel clock of 
Fig. 9.9 Pulse measurement at sample position with interferometric autocorrelation showing an approximately transform-limited pulse. Inset shows the beam profile of the laser measured with a CCD camera at the sample position with vertical and horizontal line beam profiles. From [46] Reproduced under Creative Commons Attribution License (CC BY; https:// creativecommons.org/ licenses/by/3.0/ legalcode)

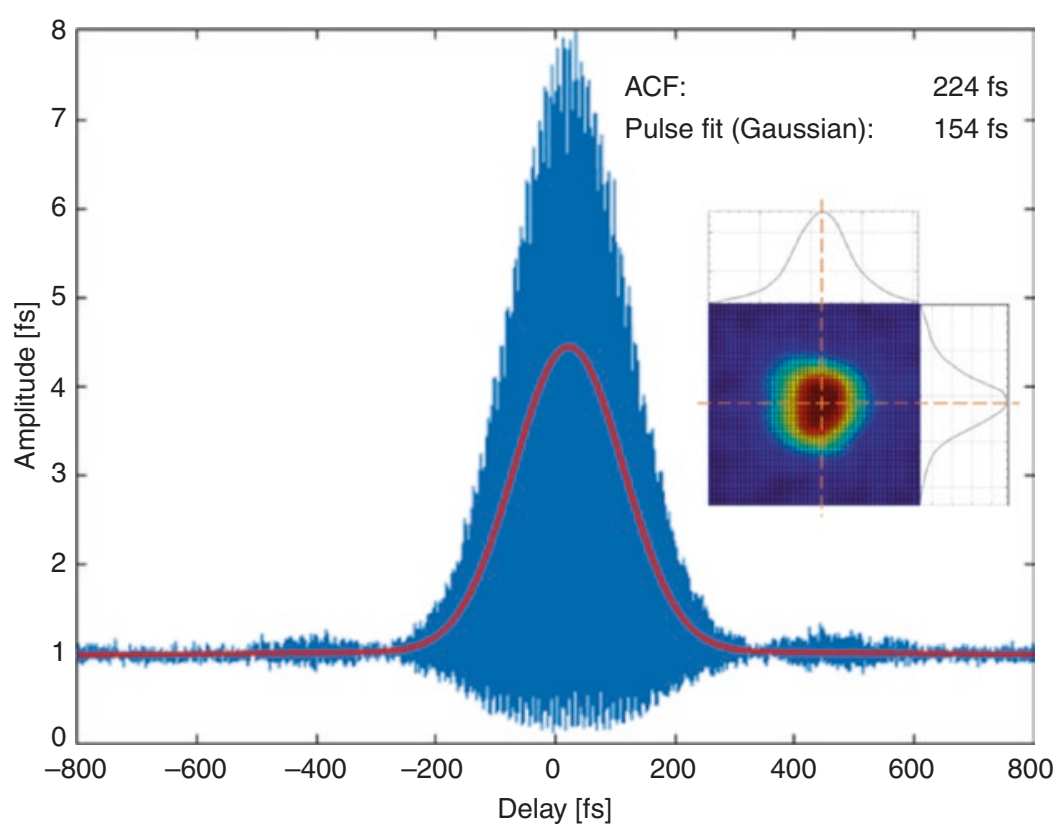

$10 \mathrm{MHz}$. Confocal reflectance images were digitized at a resolution of $768 \times 768$ pixels with a frame rate of $\sim 9 \mathrm{~Hz}$. Fluorescence images were digitized at a $256 \times 256$ pixel resolution (by binning the corresponding signal to superpixels) and were averaged over 2-3 min. Customized software for real-time eye tracking (Heidelberg Engineering) was used for imaging. The confocal reflectance image served as a reference for the two-photon fluorescence signals, whose acquisition took place after correct positioning and localization. In this manner, each detected fluorescence photon could be assigned to the corresponding pixel from the reflectance image.

Two mouse strains, Thy1-YFP-16 [B6. Cg-Tg(Thy1-YFP)16Jrs/J; 6 months old male, 35-40 g, The Jackson Laboratory, Bar Harbor, Maine] and Thy1-GCaMP3 [B6.Cg-Tg(Thy1GCaMP3)6Gfng/J; 6 months old male, 35-40 g, The Jackson Laboratory], were used for imaging. Mice were anesthetized with ketamine $(100 \mathrm{mg} /$ $\mathrm{kg})$ and xylazine $(10 \mathrm{mg} / \mathrm{kg})$ by intraperitoneal injection. Pupils were dilated with one drop of $1 \%$ tropicamide (Mydriacyl, Alcon Laboratories, Mississauga, Ontario, Canada) and one drop of 2.5\% phenylephrine (Mydfrin, Alcon Laboratories). After dilation, a 3.2-mm plano contact lens (Cantor and Nissel, Brackley, United
Kingdom) was placed on the cornea to maintain corneal hydration and compensate for most of the nonspherical refractive errors arising from the corneal surface. During imaging, the mouse was placed on a custom-built translation stage and a bite bar was used to stabilize the head for camera alignment. All experimental procedures followed the guidelines of the Canadian Council on Animal Care, and protocols were approved by the Dalhousie University Committee on Laboratory Animals.

Fluorescence lifetime maps, obtained with fluorescence lifetime imaging (FLIM), deliver additional information about cell health [48-50]. FLIM is mainly concentration independent and measures the average duration a molecule remains in an excited state. This duration is unique, providing a molecular fingerprint [51]. Changes in fluorescence lifetime reflect changes in cellular environment, such as temperature, $\mathrm{pH}$, ion, and oxygen concentration $[45,52]$.

First, we performed imaging at two different retinal depths in Thy1-YFP-16 mice. The simultaneous acquisition of the confocal reflectance image as well as the TPE image are shown in Fig. 9.10.

Focused at the level of the retinal nerve fiber layer where axons of RGCs are located, the con- 

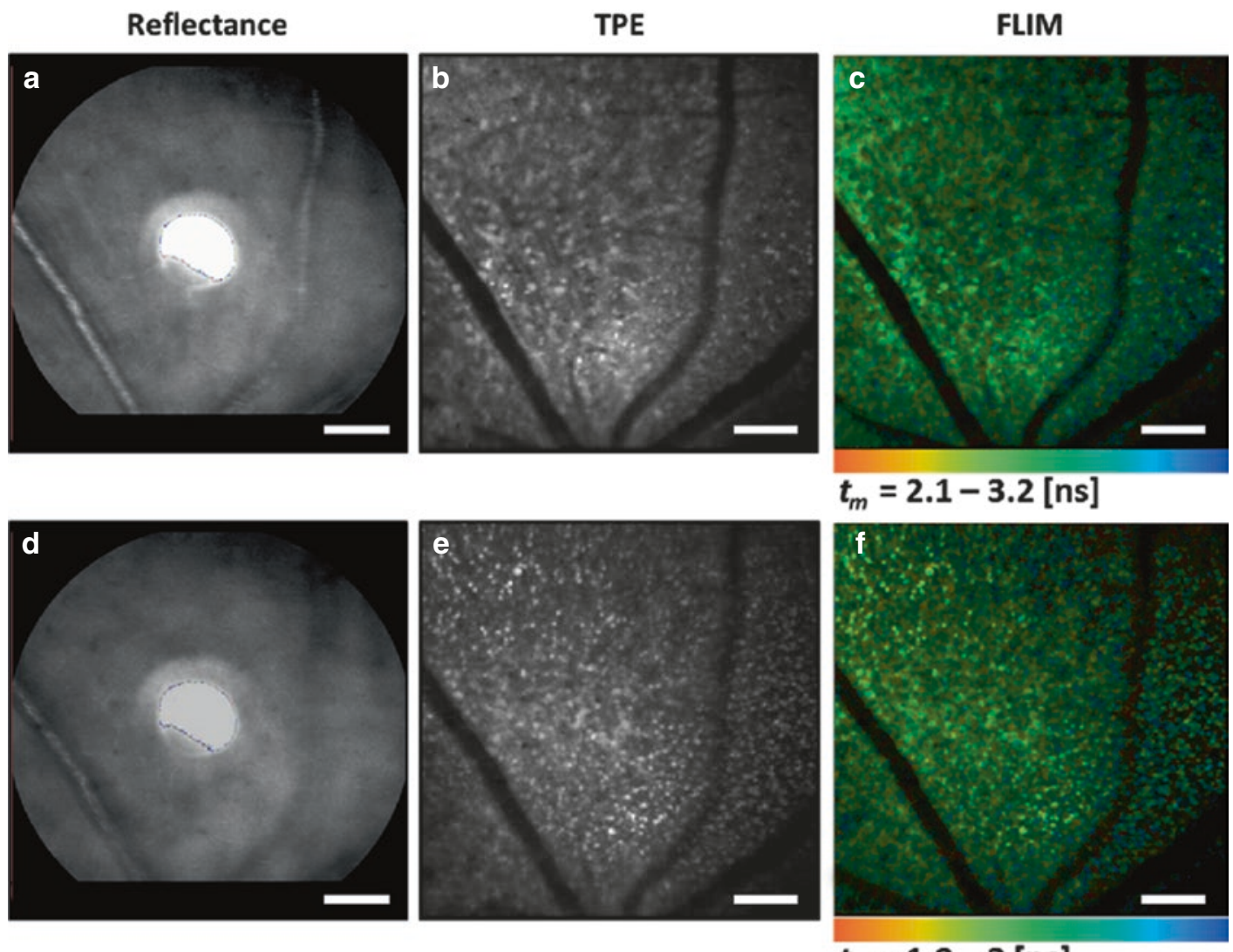

Fig. 9.10 In vivo confocal reflectance and two-photon images of the retina of a Thy1-YFP-16 mouse. (a) Confocal reflectance image showing mouse fundus. (b) Simultaneously obtained TPE fluorescence image at same transverse and axial position as in (a). (c) FLIM of (b) with scale bar of fluorescence lifetime (tm). (d) Confocal reflectance image at same transverse position as in (a) but

focal reflectance images visualize the mouse fundus (Fig. 9.10a), whereas the TPE images show RGCs (Fig. 9.10b). For each TPE pixel, the mean fluorescence lifetime (tm) was determined and displayed in a color-coded FLIM map with a decay time range from $2.1 \mathrm{~ns}$ (red) to $3.2 \mathrm{~ns}$ (blue) (Fig. 9.10c). The same imaging was performed $\sim 20-\mu \mathrm{m}$ deeper but at the same lateral position. This axial position was approximately at the level of the RGC somas (Fig. 9.10e, f). Although RGCs are clearly visualized in the Thy1-YFP-16 mouse strain (Fig. 9.10b, e), the acquisition of static fluorescence intensity measurements of RGCs does not deliver sufficient at different depths. (e) Simultaneously obtained twophoton image clearly showing RGCs. (f) FLIM of (e). Images were obtained with power levels $\sim 10 \mathrm{~mW}$ and exposure times of 2-3 min. Scale bar, $100 \mu \mathrm{m}$. From [46] Reproduced under Creative Commons Attribution License (CC BY; https://creativecommons.org/licenses/by/3.0/ legalcode)

information to discriminate functional from nonfunctional RGCs [47]. Previous work from our group has shown that, after experimental optic nerve injury, some RGCs expressing GCaMP3, a calcium indicator whose dynamics are related to changing calcium levels during neuronal action potentials, do not respond to a stimulus [19]. Dynamic fluorescence intensity imaging of these markers enables probing of cellular function of individual RGCs in response to physiologic stimuli.

Next, to determine whether imaging calcium dynamics with GCaMP3 was feasible with TPE fluorescence imaging, we imaged RGCs in the 
Thy1-GCaMP3 mouse strain. We performed imaging at two different power levels and integration times to determine if sufficient fluorescence signals could be generated to visualize individual RGCs (Fig. 9.11). Even at low-power levels, (close to the human use safety threshold) [9], individual RGCs were clearly visible. The quantity of fluorescence photons detected was too low to calculate an additional lifetime map.

FLIM measurements as presented in (Fig. 9.10c, f) have the potential to provide critical and complementary information about cell status [31, 53] and differentiate among RGC sub-
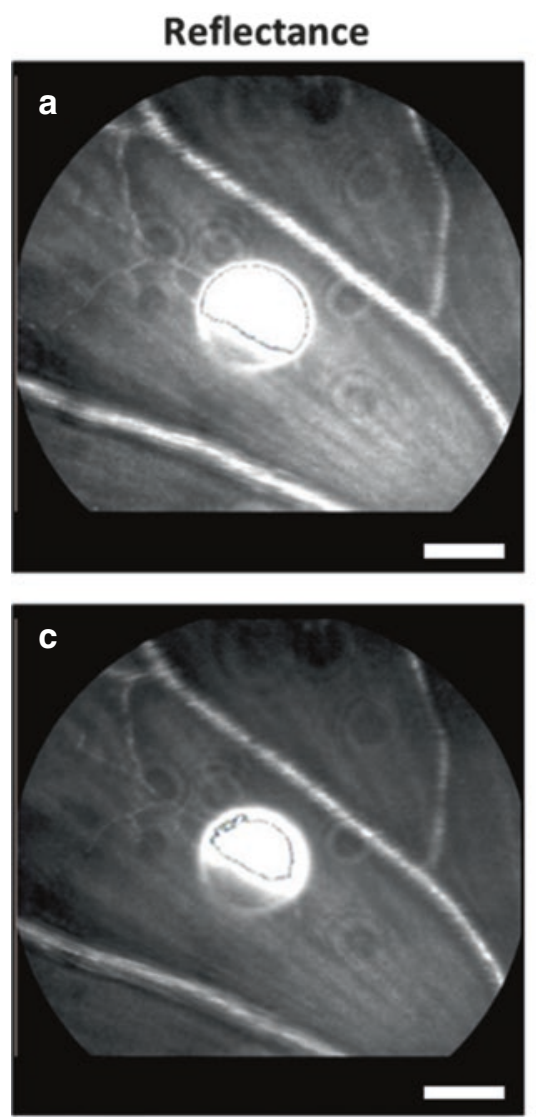

Fig. 9.11 In vivo confocal reflectance and TPE fluorescence images of the retina of a Thy1-GCaMP3 mouse at different power levels and integration times. (a) Confocal reflectance image showing the mouse fundus with $\sim 10$ $\mathrm{mW}$ laser power. (b) Two-photon image acquired simultaneously with (a) showing individual GCaMP3 expressing RGCs; power $\sim 10-\mathrm{mW}$ and $\sim 3$-min exposure time. (c) and types with different levels of vulnerability to damage. This work is currently in progress.

\subsection{Future Application of Two-Photon Scanning Laser Ophthalmoscopy}

Currently, most experimental models of diseases leading to RGC loss, such as glaucoma and ischemic optic neuropathy rely on qualitative or quantitative assessment of RGC loss after tissues have been processed after termination of the experi-
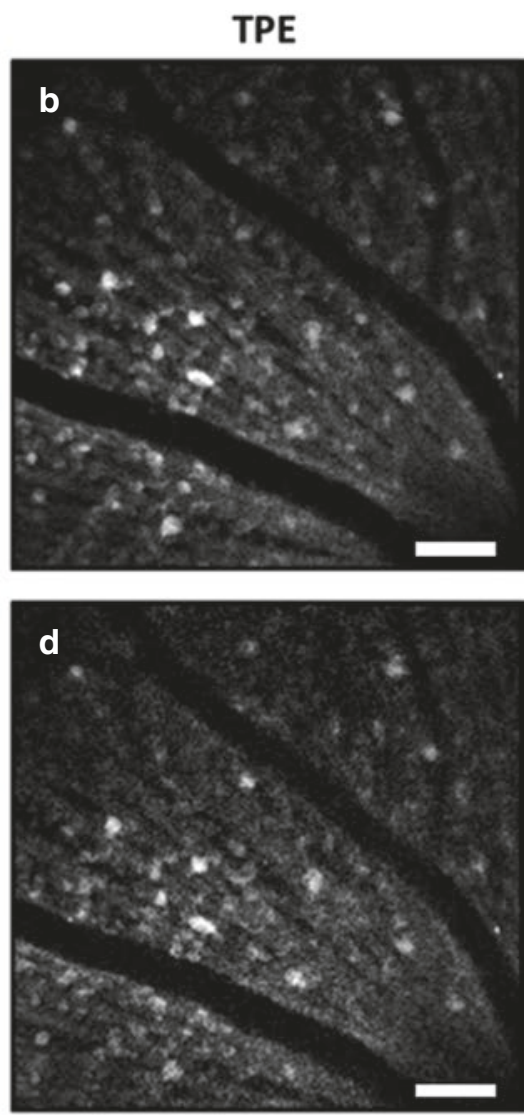

(d) Reflectance and two-photon images, respectively, at the same lateral and axial location as (a) but with lower laser power $(\sim 3 \mathrm{~mW})$; exposure time $\sim 3.5 \mathrm{~min}$. Scale bar, $50 \mu \mathrm{m}$. From [46] Reproduced under Creative Commons Attribution License (CC BY; https://creativecommons.org/ licenses/by/3.0/legalcode) 
ment. Therefore, a longitudinal assessment of the degree or rate of RGC loss is not typically made in vivo, resulting in a limitation in the assessment of damage in chronic disease models.

The availability of transgenic mice that express fluorophores under the control of promoters thought to be expressed by RGCs, such as Thy1 [54], have greatly advanced the field. Several examples of characterization of RGC loss in vivo after experimental optic nerve diseases have been published $[14,15]$. While readily available, these transgenic strains pose some limitations given that the expression profile of the promoters change after injury and encapsulation of the fluorophore by phagocytosing cells after RGC death. Hence, the specificity and accuracy of RGC loss with these strains can limit the accuracy of experiments. Even if attempts to move away from transgenic animals to make these applications more translatable, these problems exist even if the fluorophore is introduced exogenously via techniques such as virus-based transfection [17].

However, as recent evidence shows [19], structural fluorescent markers are likely inadequate markers of RGC integrity since the presence of fluorescence does not necessary indicate functional viability. The ability to dynamically image fluorescence after a light stimulus represents a significant advance. As this chapter has indicated, two-photon scanning laser ophthalmoscopy offers a real potential to scientists to study the functional impact of diseases causing RGC loss in experimental disease models. This technique also offers a powerful assay to study the impact of therapeutics.

Ultimately, if fluorescent indicators of functional activity can be safely introduced into RGCs in humans and safely imaged after visual stimulation with two-photon scanning laser ophthalmoscopy, remarkable progress would be made in the diagnosis and treatment of many ocular diseases. Indeed, single cell functional imaging of RGCs could represent one of the single-most important imagining innovations.

\section{References}

1. Denk W, Strickler JH, Webb WW. Two-photon laser scanning fluorescence microscopy. Science. 1990;248:73-6.

2. Helmchen F, Denk W. Deep tissue two-photon microscopy. Nat Methods. 2005;2:932-40.

3. Svoboda K, Yasuda R. Principles of two-photon excitation microscopy and its applications to neuroscience. Neuron. 2006;50:823-39.

4. Imanishi Y, Lodowski KH, Koutalos Y. Two-photon microscopy: shedding light on the chemistry of vision ${ }^{\dagger}$. Biochemistry. 2007;46:9674-84.

5. Dillon J, Zheng L, Merriam JC, Gaillard ER. Transmission spectra of light to the mammalian retina. Photochem Photobiol. 2000;71:225-9.

6. Gray DC, et al. In vivo fluorescence imaging of primate retinal ganglion cells and retinal pigment epithelial cells. Opt Express. 2006;14:7144-58.

7. Morgan JIW, et al. Light-induced retinal changes observed with high-resolution autofluorescence imaging of the retinal pigment epithelium. Invest Ophthalmol Vis Sci. 2008;49:3715-29.

8. Benninger RKP, Piston DW. Two-photon excitation microscopy for the study of living cells and tissues. In: Bonifacino JS, Dasso M, Harford JB, LippincottSchwartz J, Yamada KM, editors. Current protocols in cell biology. Hoboken: John Wiley \& Sons, Inc; 2013. https://doi.org/10.1002/0471143030.cb0411s59.

9. Bar-Noam AS, Farah N, Shoham S. Correction-free remotely scanned two-photon in vivo mouse retinal imaging. Light Sci Appl. 2016;5:e16007.

10. Kolb H. How the Retina Works: Much of the construction of an image takes place in the retina itself through the use of specialized neural circuits. Am Sci. 2003;91:28-35.

11. Masland RH. The Neuronal Organization of the Retina. Neuron. 2012;76:266-80.

12. Baden $\mathrm{T}$, et al. The functional diversity of retinal ganglion cells in the mouse. Nature. 2016;529: 345-50.

13. Chauhan BC, et al. Longitudinal in vivo imaging of retinal ganglion cells and retinal thickness changes following optic nerve injury in mice. PLoS One. 2012;7:e40352.

14. Wang X, Archibald ML, Stevens K, Baldridge WH, Chauhan BC. Cyan fluorescent protein (CFP) expressing cells in the retina of Thy1-CFP transgenic mice before and after optic nerve injury. Neurosci Lett. 2010;468:110-4.

15. Leung CK, et al. Long-term in vivo imaging and measurement of dendritic shrinkage of retinal ganglion cells. Invest Opthalmol Vis Sci. 2011;52:1539.

16. Smith CA, Chauhan BC. Imaging retinal ganglion cells: Enabling experimental technology for clinical application. Prog Retin Eye Res. 2015;44:1-14. 
17. Smith CA, Chauhan BC. In vivo imaging of adenoassociated viral vector labelled retinal ganglion cells. Sci Rep. 2018;8:1490.

18. Chen $\mathrm{Q}$, et al. Imaging neural activity using Thy $1-$ GCaMP transgenic mice. Neuron. 2012;76:297-308.

19. Blandford SN, et al. Retinal characterization of the Thy1-GCaMP3 transgenic mouse line after optic nerve transection. Invest Opthalmol Vis Sci. 2019;60:183.

20. Yin L, et al. Imaging light responses of retinal ganglion cells in the living mouse eye. J Neurophysiol. 2013;109:2415-21.

21. Yin L, et al. Imaging light responses of foveal ganglion cells in the living macaque eye. J Neurosci. 2014;34:6596-605.

22. Zipfel WR, Williams RM, Webb WW. Nonlinear magic: multiphoton microscopy in the biosciences. Nat Biotechnol. 2003;21:1369.

23. Hunter JJ, et al. Images of photoreceptors in living primate eyes using adaptive optics two-photon ophthalmoscopy. Biomed Opt Express. 2011;2:139.

24. Palczewska G, Golczak M, Williams DR, Hunter JJ, Palczewski K. Endogenous fluorophores enable twophoton imaging of the primate eyetwo-photon imaging of the primate eye. Invest Ophthalmol Vis Sci. 2014;55:4438-47.

25. Sharma R, et al. In vivo two-photon fluorescence kinetics of primate rods and cones. Invest Opthalmol Vis Sci. 2016;57:647.

26. Sharma R, et al. Formation and clearance of all- trans -retinol in rods investigated in the living primate eye with two-photon ophthalmoscopy. Invest Opthalmol Vis Sci. 2017;58:604.

27. Rossi EA, et al. Imaging individual neurons in the retinal ganglion cell layer of the living eye. Proc Natl Acad Sci. 2017;114:586-91.

28. Akerboom J, et al. Optimization of a GCaMP calcium indicator for neural activity imaging. J Neurosci. 2012;32:13819-40.

29. Spiess E, et al. Two-photon excitation and emission spectra of the green fluorescent protein variants ECFP, EGFP and EYFP. J Microsc. 2005;217:200-4.

30. https://www.thermofisher.com/de/de/home/references/molecular-probes-the-handbook.html.

31. Dysli C, et al. Fluorescence lifetime imaging ophthalmoscopy. Prog Retin Eye Res. 2017;60:120-43.

32. Huang S, Heikal AA, Webb WW. Two-photon fluorescence spectroscopy and microscopy of NAD(P)H and flavoprotein. Biophys J. 2002;82:2811-25.

33. Breunig HG, Studier H, König K. Multiphoton excitation characteristics of cellular fluorophores of human skin in vivo. Opt Express. 2010;18:7857.

34. Miura Y, et al. Two-photon microscopy and fluorescence lifetime imaging of retinal pigment epithelial cells under oxidative stress. Invest Opthalmol Vis Sci. 2013;54:3366.
35. Schmid PH. at de wikipedia, edited by Marc Gabriel. Deutsch: Schichten und Zelltypen der Säugetierretina. 2007.

36. Kojima D, et al. UV-sensitive photoreceptor protein OPN5 in humans and mice. PLoS One. 2011;6:e26388.

37. Mondal PP, Diaspro A. Fundamentals of fluorescence microscopy: exploring life with light. Dordrecht: Springer; 2014.

38. Kubitscheck U, editor. Fluorescence microscopy: from principles to biological applications. Hoboken: Wiley-Blackwell; 2013.

39. Göppert-Mayer M. Über Elementarakte mit zwei Quantensprüngen. Ann Phys. 1931;401:273-94.

40. Xu C, Webb WW. Measurement of two-photon excitation cross sections of molecular fluorophores with data from 690 to $1050 \mathrm{~nm}$. J Opt Soc Am B. 1996;13:481.

41. Brakenhoff GJ, Müller M, Ghauharali RI. Analysis of efficiency of two-photon versus single-photon absorption for fluorescence generation in biological objects. J Microsc. 1996;183:140-4.

42. Diaspro A, et al. Multi-photon excitation microscopy. Biomed Eng Online. 2006;14:5-36.

43. Wang W, Liu Y, Xi P, Ren Q. Origin and effect of high-order dispersion in ultrashort pulse multiphoton microscopy in the $10 \mathrm{fs}$ regime. Appl Optics. 2010;49:6703.

44. Xu C, Zipfel W, Shear JB, Williams RM, Webb WW. Multiphoton fluorescence excitation: new spectral windows for biological nonlinear microscopy. Proc Natl Acad Sci. 1996;93:10763-8.

45. Horilova J, et al. Fingerprinting of metabolic states by $\mathrm{NAD}(\mathrm{P}) \mathrm{H}$ fluorescence lifetime spectroscopy in living cells: a review. Med Photonics. 2015;27:62-9.

46. Kamali T, et al. Simultaneous in vivo confocal reflectance and two-photon retinal ganglion cell imaging based on a hollow core fiber platform. J Biomed Opt. 2018;23:1.

47. Langenegger SJ, Funk J, Töteberg-Harms M. Reproducibility of retinal nerve fiber layer thickness measurements using the eye tracker and the retest function of spectralis SD-OCT in glaucomatous and healthy control eyes. Invest Ophthalmol Vis Sci. 2011;52:3338-44.

48. Bastiaens PIH, Squire A. Fluorescence lifetime imaging microscopy: spatial resolution of biochemical processes in the cell. Trends Cell Biol. 1999;9:48-52.

49. Becker W. Fluorescence lifetime imaging - techniques and applications. J Microsc. 2012;247: 119-36.

50. Becker W, et al. Fluorescence lifetime imaging by time-correlated single-photon counting. Microsc Res Tech. 2004;63:58-66.

51. Berezin MY, Achilefu S. Fluorescence lifetime measurements and biological imaging. Chem Rev. 2010;110:2641-84. 
52. Suhling $\mathrm{K}$, et al. Fluorescence lifetime imaging (FLIM): Basic concepts and some recent developments. Med Photonics. 2015;27:3-40.

53. Walsh AJ, Sedelnikova A, Tolstykh GP, Ibey BL, Beier HT. Fluorescence lifetime imaging of calcium flux in neurons in response to pulsed infrared light. In: 10069, 100691B-10069-8. 2017.

54. Feng G, et al. Imaging neuronal subsets in transgenic mice expressing multiple spectral variants of GFP. Neuron. 2000;28:41-51.

Open Access This chapter is licensed under the terms of the Creative Commons Attribution 4.0 International License (http://creativecommons.org/licenses/by/4.0/), which permits use, sharing, adaptation, distribution and reproduction in any medium or format, as long as you give appropriate credit to the original author(s) and the source, provide a link to the Creative Commons license and indicate if changes were made.

The images or other third party material in this chapter are included in the chapter's Creative Commons license, unless indicated otherwise in a credit line to the material. If material is not included in the chapter's Creative Commons license and your intended use is not permitted by statutory regulation or exceeds the permitted use, you will need to obtain permission directly from the copyright holder. 